\title{
Spatial relationship between the c-Fos distribution and enkephalinergic, substance $P$, and tyrosine hydroxylase innervation fields after acute treatment with neuroleptics olanzapine, amisulpride, quetiapine, and aripiprazole in the rat septum
}

\author{
Alexander Kiss, Jana OsackA \\ Institute of Experimental Endocrinology, Biomedical Research Center, Slovak Academy of Sciences, \\ Bratislava, Slovak Republic \\ E-mail: alexander.kiss@savba.sk
}

Objective. The aim of the present study was to demonstrate the spatial relationship between the c-Fos immunoreactive cells elicited by an acute treatment with neuroleptics including amisulpride (AMI), olanzapine (OLA), quetiapine (QUE), and aripiprazole (ARI) and enkephalinergic (ENK), substance $\mathrm{P}(\mathrm{SP})$, and tyrosine hydroxylase (TH) innervation fields in the rat septum.

Methods. Male Sprague Dawley rats received a single injection of OLA (5 mg), ARI (10 mg), AMI $(20 \mathrm{mg})$, QUE (15 mg/kg/b.w.). Ninety min after antipsychotics administration, the animals were transcardially perfused with a fixative and the brains cryocut into serial coronal sections of $35 \mu \mathrm{m}$ thickness. The sections were processed for c-Fos staining using an avidin-biotin-peroxidase complex and visualized by nickel intensified diaminobenzidine to reach black endproduct. Afterwards, the sections were exposed to ENK, SP, and TH antibodies and the reaction product visualized by biotin-labeled fluorescent AlexaFluor 564 dye. The data were evaluated from the sections either simultaneously illuminated with fluorescent and transmission microscope beams or after merging the separately illuminated sections in the Adobe Photoshop 7.0 software.

Results. ENK, SP, and TH displayed characteristic spatial images formed by a dense accumulation of immunoreactive fibers and terminals on the both sides of the septum. A dense plexus of axons formed by ENK and SP immunopositive terminals were situated predominantly in the lateral, while TH ones more medial portion of the septum. QUE and AMI activated distinct amount of c-Fos expression in cells located within the SP-immunoreactive principal innervation field. The OLA effect on the c-Fos expression was very pronounced in the ventral TH-labeled principal innervation field including the space between the ENK field ventral portion and the dorsal margin of the accumbens nucleus shell. Generally, the occurrence of c-Fos cells in the ENK-immunoreactive principal innervation field, in comparison with the surrounding septal area, was less abundant after all of the four antipsychotics treatments.

Conclusion. The data of the present study indicate that ENK, SP, and TH innervation fields may influence separate populations of septal cells activated by AMI, OLA, QUE, and ARI and that each of these region-differently innervated cells may be associated with the functional heterogeneity of the individual lateral septal nuclei.

Key words: amisulpride, olanzapine, quetiapine, aripiprazole, enkephalin, substance P, tyrosine hydroxylase, c-Fos, immunohistochemistry, rat

Corresponding author: Dr. Alexander Kiss, PhD., D.Sci., Institute of Experimental Endocrinology, Biomedical Research Center, Slovak Academy of Sciences, Dubravska cesta 9, 84505 Bratislava, Slovak Republic; phone: 004212532295225; e-mail: ueenkiss@savba.sk. 
The septum (SEP) is a forebrain area occupying the brain middle portion delineated by the corpus callosum from the top and the lateral brain ventricles from the sides. It is composed of medial and lateral septal nuclei. The lateral septal nucleus can be divided into dorsal, intermediate and ventral parts (Swanson and Cowan 1979). It receives its major input from the hippocampal formation and projects to the medial septal-diagonal band complex. The medial septal nucleus/diagonal band complex projects back to the hippocampal formation. Actually, the septum forms reciprocal linkages with a number of brain structures including the olfactory bulb, hippocampus, amygdala, hypothalamus, midbrain, habenula, cingulate gyrus, and thalamus (Swanson and Cowan 1979).

The SEP is an important component of the limbic system that may mediate some of the therapeutic actions of antipsychotics (Robertson and Fibiger 1996). However, the anatomical composition of the septal nuclei is quite complicated, divided into many subareas, whereas different substances may differently affect the cells in the individual subareas (Risold and Swanson 1997; Swanson and Risold 2000). It has been demonstrated that the SEP is a target of many antipsychotics including olanzapine (Robertson and Fibiger 1996; Sebens et al. 1998), amperozide (Nomikos et al. 1997), asenapine (Majercikova et al. 2014), haloperidol and clozapine (MacGibbon et al. 1994), and risperidon (Wan et al. 1995). As indicated by the presence of c-Fos expression, greatest population of septal cells affected by antipsychotics is situated in the lateral septum intermediate and the ventral parts, which have been cytoarchitectonally defined along the whole dorsoventral axis of the brain (Swanson and Cowan 1979).

Olanzapine (OLA), amisulpride (AMI), and quetiapine (QUE) belong to the second, while aripiprazole (ARI) the third generation of atypical antipsychotics (Goodnick and Jerry 2002; Winans 2003; Mailman and Murthy 2010). All of them are mainly used in the treatment of schizophrenia (Tamminga and Kane 1997; Ozdemir et al. 2002; Grady et al. 2003; Swainston-Harrison and Perry 2004; Natesan et al. 2011; de Bartolomeis et al. 2013, 2015). A common mechanism of the atypical antipsychotics action is the dopamine (DA) receptors blockade, but their affinity to DA receptors and other pharmacological characteristics differs substantially. OLA is mostly an antagonist of serotoninergic 5-HT2A receptors, but it also may antagonize dopaminenergic D2 and serotonergic 5-HT2C-6, adrenergic al, and muskarinic M1 receptors. OLA induces metabolic side effects like weight gain and type 2 diabetes. AMI has a high-affinity for dopaminergic D2/D3, but not serotonergic and other types of receptors (Natesan et al. 2008). In lower doses, AMI by blocking dopaminergic D2/D3 autoreceptors improves the negative symptoms of schizophrenia, while in higher doses by blocking the post-synaptic DA receptors reduces the positive symptoms (Natesan et al. 2008). AMI is devoided of affinity to other sorts of receptors (Schoemaker et al. 1997). QUE is a powerful sedative exhibiting high affinity for serotoninergic 5-HT2 and dopaminergic D2 receptors. ARI, representing the 3rd generation of antipsychotics, has not only an antagonistic effect on dopamine D2 and serotonergic 5-HT2A receptors, but also acts as a partial highefficacy agonist on the other D2 receptor subpopulations and 5-HT1A receptors (Swainston-Harrison and Perry 2004). ARI may also affect D3, adrenergic $\alpha 1$, and histaminergic $\mathrm{H} 1$ receptors and reduce the susceptibility to metabolic syndrome. OLA, QUE, and ARI are lack of significant extrapyramidal side effects (EPS) (Nemeroff et al. 2002; Natesan et al. 2006). However, AMI induces not only EPS but also a distinct hyperprolactinemia (Goodnick et al. 2002; Mandal et al. 2014).

It has also been demonstrated that the septum is heavily innervated by a number of chemically well-defined neural fiber networks, which may be involved in the modulation of the antipsychotic's actions. Dense plexus of axons occupying the lateral SEP has been demonstrated by immunostaining for enkephalin (Onteniente et al. 1989), substance P (Gall and Moore 1984; Morita et al. 1991), neurotensin (Kohler and Eriksson 1984), tyrosine hydroxylase (Gall and Moore 1984; Jakab and Leranth 1993), somatostatin (Kohler and Eriksson 1984; Jakab and Leranth 1993), corticotropin-releasing hormone (Sakanaka et al. 1988), calcitonin generelated peptide (Sakanaka et al. 1985), cholecystokinin (Hokfelt et al. 1980), thyrotropin-releasing hormone (Ishikawa et al. 1984), vasopressin and oxytocin (deVries and Buijs 1983), etc. In spite of the number of studies performed, the spatial relationship between the septal neurons activated by antipsychotics and the surrounding innervation plexuses of different chemical composition has not been demonstrated yet.

The aim of the present study was to anatomically characterize and delineate the spatial interrelationship between the c-Fos distribution induced by a single treatment with olanzapine, amisulpride, quetiapine, and aripiprazole, and the ENK, SP, and TH innervation fields in the rat septum. 


\section{Materials and methods}

Animals. Adult male Sprague Dawley rats $(n=42)$ purchased in Velaz (Prague, Cuech Republic) weighing 280-300 g were used. They were housed three or four per cage in a room with controlled temperature $\left(22 \pm 1^{\circ} \mathrm{C}\right)$, light (12-hour light/dark cycle with lights on at 06:00 h), and humidity (55\%). Animals were provided with a regular rat chow (dry pellets) and tap water ad libitum. Principles of the laboratory animal care and the experimental procedures used were approved by the State Veterinary and Food Administration of the Slovak Republic Committee (Aproval protocol number 1461/17-221) and the investigation conditions were in accordance with the guidelines with the National Institute of Health Guide for the Care and Use of Laboratory Animals (NIH Publications No. 80-23) revised 1996 guidelines for the Care and Use of Laboratory Animals published by the U.S. National Institutes of Health.

Experimental groups. The rats were divided into 5 groups: 1) vehicle controls (VEH, $\mathrm{n}=5$ ), 2) olanzapine (OLA, $n=6)$, 3) amisulpride (AMI, $n=6)$, 4) quetiapine (QUE, $\mathrm{n}=6$ ), and 5) aripiprazole (ARI, $\mathrm{n}=7$ ).

Antipsychotics treatments. Animals were injected intraperitoneally with vehicle or a particular antipsychotic. VEH group was injected with $4 \%$ DMSO dissolved in saline. The antipsychotic-treated animals received the following doses of the drugs: OLA $5 \mathrm{mg} / \mathrm{kg}$, AMI $20 \mathrm{mg} / \mathrm{kg}$, QUE $15 \mathrm{mg} / \mathrm{kg}$, and ARI $10 \mathrm{mg} / \mathrm{kg}$ of the body weight (b.w.). All the antipsychotics were dissolved in 4\% DMSO in saline.

Perfusion procedure. Ninety min after the treatments (the time of the antipsychotics duration was selected based on a pilot study, in which c-Fos expression extensiveness was tested 90, 120, and 150 min of post-antipsychotics treatment), the rats were anesthetized by a combined treatment of Zoletil (30 mg/kg, Virbac, Carros, France) and Xylariem (15 mg/kg, Riemser Germany) in the volumes $0.1 \mathrm{ml}$ and $0.24 \mathrm{ml} / 300 \mathrm{~g}$ b.w., respectively and sacrificed by a transcardial perfusion with $60 \mathrm{ml}$ of saline containing $450 \mu \mathrm{l}$ of heparin (5000 IU/l, Zentiva, Slovakia) followed by $250 \mathrm{ml}$ of fixative containing $4 \%$ paraformaldehyde (Sigma-Aldrich, Germany) in $0.1 \mathrm{M}$ phosphate buffer (PB, pH 7.4). The brains were postfixed in a fresh fixative overnight, washed two times in $0.1 \mathrm{M} \mathrm{PB}$, infiltrated with $30 \%$ sucrose for 2 days at $4{ }^{\circ} \mathrm{C}$, cut into $30 \mu \mathrm{m}$ thick coronal sections using Reichert-Jung, cryo-cut E (Austria), and collected in a cryoprotectant solution at $-20^{\circ} \mathrm{C}$ until used.

c-Fos immunohistochemistry. One set of freefloating sections was repeatedly washed in cold $0.1 \mathrm{M}$
$\mathrm{PB}$ and preincubated in a blocking solution of $0.3 \%$ $\mathrm{H}_{2} \mathrm{O}_{2}$ in $0.1 \mathrm{M}$ PB (Fisher Scientific, Fair Lawn, NJ, USA) for $20 \mathrm{~min}$ at room temperature (RT). Then the sections were rinsed $3 \times 10 \mathrm{~min}$ in $0.05 \mathrm{M} \mathrm{PB}$ and incubated with a polyclonal c-Fos antiserum (12-5) diluted 1:1500 in $0.05 \mathrm{M} \mathrm{PB}$ containing 4\% normal goat serum (Gibco, Grand Island, NY, USA), 0.5\% Triton X-100 (Koch-Light Lab. Ltd., Colnbrook Berks, England), and $0.1 \%$ sodium azide (Sigma Chemical Ltd. St. Louis MO, USA) for $48 \mathrm{~h}$ at $4{ }^{\circ} \mathrm{C}$. After several rinsing in $\mathrm{PB}$, the sections were incubated with biotinylated goat anti-rabbit IgG (1:500, VectorStain Elite ABC Kit, Vector Lab., Burlingame, CA, USA) in PB for 90 min at RT. Next PB rinsing was followed by incubation with the avidin-biotin peroxidase complex (1:250) for $90 \mathrm{~min}$ at RT. After several washings in $0.05 \mathrm{M}$ sodium acetate buffer (SAB, pH 6.0), c-Fos antigenic sites were visualized by nickel-enhanced 3,3'-diaminobenzidine tetrahydrochloride $(0.0625 \%$ DAB, $2.5 \%$ nickel chloride, SigmaAldrich, No. 7718-54-9), in SAB containing 0.0006\% hydrogen peroxide. Developing time was 6-10 min. The heavy metal-intensification of DAB yielded black staining in the Fos labeled nuclei.

Enkephaline, substance $P$, and tyrosine hydroxylase immunohistochemistry. In the three set of c-Fos labeled sections, counterstaining with ENK, SP, and $\mathrm{TH}$ antibodies was performed. After several washings in the $0.05 \mathrm{M} \mathrm{PB}$, the c-Fos containing sections were exposed to ENK (mouse monoclonal), SP (rabbit polyclonal), and TH (rabbit monoclonal) antibodies for 2 days at the RT. Then the sections were washed 3 times in the $0.05 \mathrm{M} \mathrm{PB}$ and incubated with secondary goat anti-mouse Alexa Fluor 568 (ab150078) and goat-anti rabbit Alexa Fluor 568 antibodies dissolved 1:250 in the 0.05 M PB containing $0.1 \%$ Triton X 100 in $0.05 \mathrm{M}$ PB. After overnight incubation with the secondary antibodies the sections were well washed in the $0.05 \mathrm{M} \mathrm{PB}$ and mounted on positive charged adhesive slides, dried at RT for 120 min, coverslipped with Pertex (Stockholm, Sweden), and stored in dark histological boxes. The antibodies specificity tests were performed by omission of the primary antibody in the immunohistochemical procedure.

Brain area investigated. The dorsal (seDL), the intermediate (seIM) and the ventral (seVL) parts of the lateral septum have been investigated and anatomically designated between bregma 1.00 and 0.48 coordinates, based on the anatomical atlas of Paxinos and Watson (1997). Photomicrographs (4-6 sections/structure), were captured on Axio-Imager A1 light-fluorescent microscope (Carl Zeiss) coupled to a video camera and monitor. 
Antibodies. The primary polyclonal rabbit antic-Fos antibody was a gift (see acknowledgement). Monoclonal enkephalin (ab150346) and tyrosine hydroxylase (ab137869) and polyclonal substance $\mathrm{P}$ (ab67006), antibodies as well as the secondary goat anti-mouse Alexa Fluor 568 (ab175473) and goat-anti rabbit Alexa Fluor 568 (ab175471) antibodies were purchased from the Abcam (Cambridge UK).

Data evaluation. The microscopic pictures were captured using Axio-Imager A1 light microscope (Carl Zeiss) coupled to a video camera and monitor either under a parallel illumination of the section with fluorescent and transmission beams or separated illuminations. In the latter case, the c-Fos staining was captured using transmission beams, while ENK, $\mathrm{SP}$, and $\mathrm{TH}$ fluorescent images by the fluorescent beams. The images obtained by the separated light and fluorescent beams, were merged and evaluated in the Adobe Photoshop 7.0 software.

\section{Results}

Enkephaline, tyrosine hydroxylase, and substance $\mathbf{P}$ innervation fields. Neither of the three substances studied (ENK, TH, SP) created homogeneous innervation over the whole septum. Besides of their irregularly dispersed immunoreactive fiber fragments in the septum, each of them displayed principal and characteristic spatial images (Figure 1) formed by a dense accumulation of immunoreactive fibers and terminals arranged bilaterally, i.e. on the both sides of the septum. Although ENK, SP, and TH agglomerations appeared to be clearly separated from each other (Figure 1), some of their marginal parts showed a pattern of mutual overlapping (Figure 1).

The principal ENK-immunoreactive agglomeration was formed by a dense plexus of axons and terminals in the dorsal, intermediate, and ventral parts of the septum. The ENK-containing immunoreactive plexus was represented by a number of neuronal fibers and terminals forming several heterogeneous formations, i.e. subfields, near the lateral ventricle. Generally, four subfields were distinguished in the ENK-immunoreactive plexus zone: ventrolateral (VLat), dorsolateral (DLat), ventromedial (VMed), and dorsomedial (DMed) one (Figure 2A). The VLat and DLat subfields were densely packed with the neuronal fibers and terminals. The VLat subregion run parallelly with the lateral ventricle wall, while the DLat one gradually deviated medially. The VMed and DMed ENK subfields were less densely packed with the ENK-containing immunoreactive neuronal fibers and terminals. In the latter two subfields, ENKcontaining immunoreactive axons formed distinct
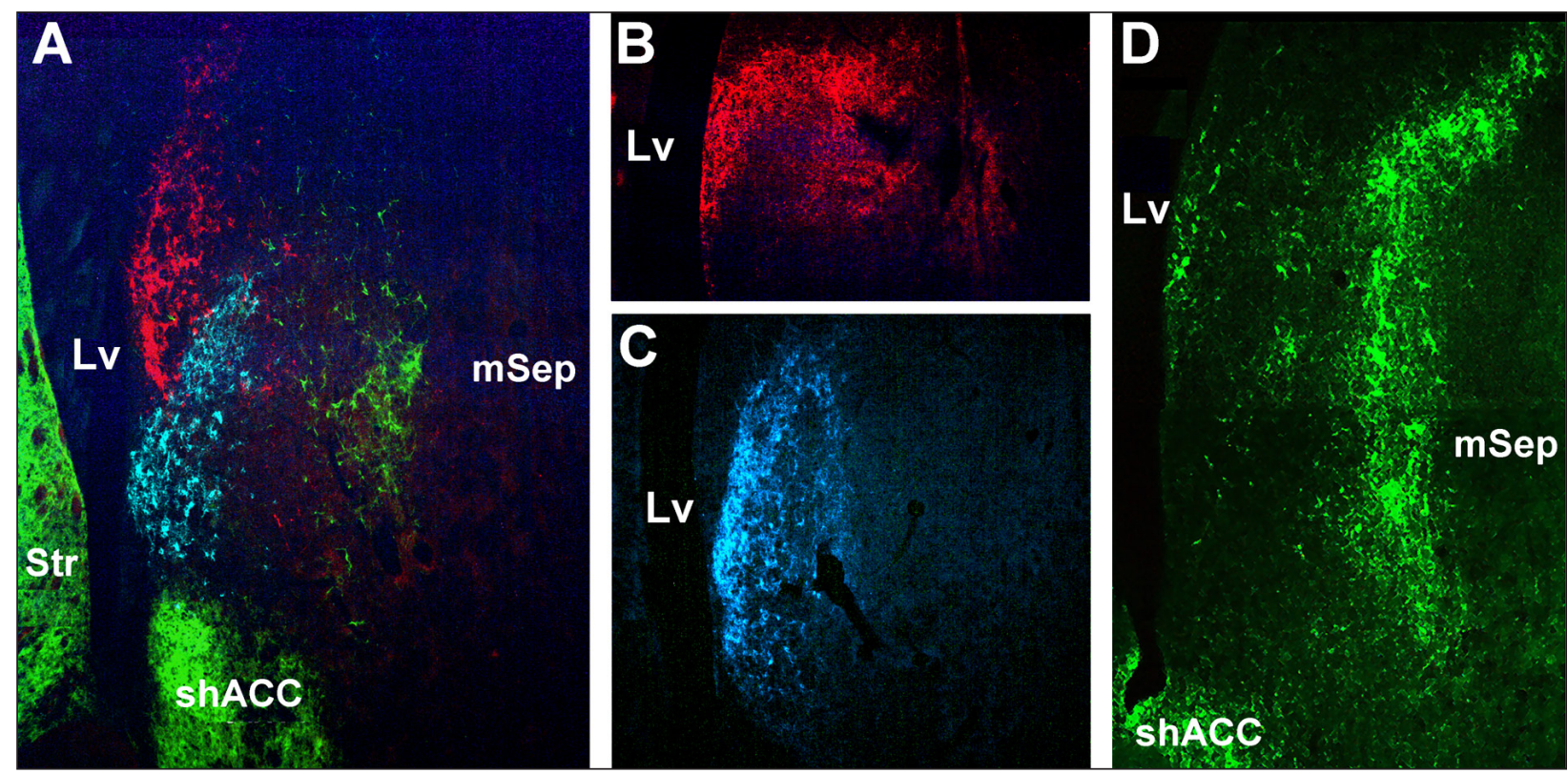

Figure 1. Schematic illustration of the spatial disposition of the enkephalinergic (ENK), substance P (SP), and tyrosine hydroxylase $(\mathrm{TH})$ principal innervation fields in the rat septum (A). Separate pictures (B, C, D) indicate the shape in the maximal dimensions of the individual principal ENK, SP, and TH innervation fields. Abbreviations: blue - ENK innervation field; red - SP innervation field; green - TH innervation field; Lv - lateral ventricle; mSEP - medial septum; shACC - accumbens nucleus shell; Str - striatum. 


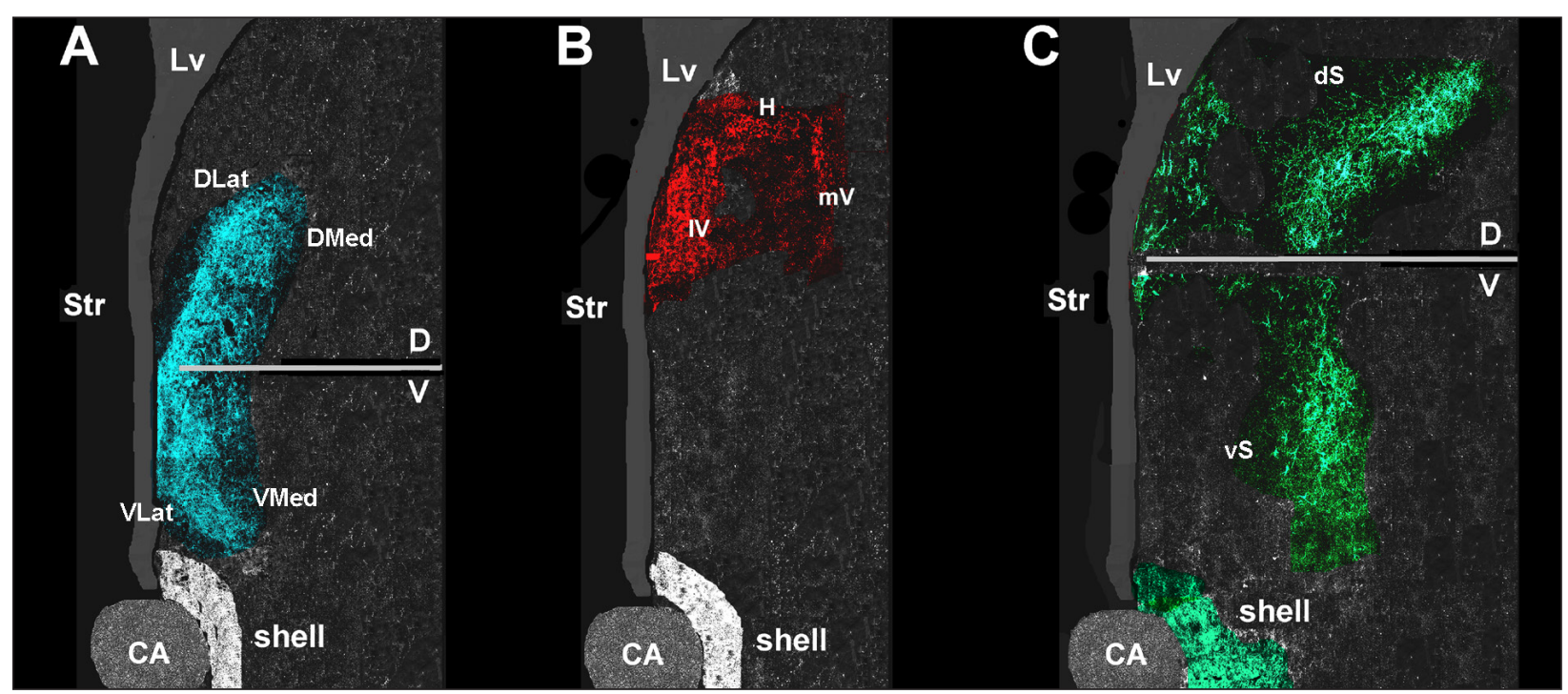

Figure 2. Schematic illustration of the enkephalinergic (ENK), substance P (SP), and tyrosine hydroxylase (TH) principal innervation fields subfields in the rat septum. (A) - demonstrates the ENK 4 subfields (DLat - dorsolateral, DMed - dorsomedial, VLat ventrolateral, VMed - ventromedial); (B) - the SP 3 subfields (IV - lateral vertical, $\mathrm{mV}$ - medial vertical, $\mathrm{H}$ - horizontal); (C) - the $2 \mathrm{TH}$ subfields (vS - ventral vertical, dS - dorsal medially twisted). Abbreviations: CA - commissure anterior; (D) - dorsal level; V - ventral level; Lv - lateral ventricle; shell - accumbens n. shell; Str - striatum.

pericellular and peridendritic terminal arbors and baskets scattered around the ventral septal nucleus neurons. From the VLat subfield many of the ENKimmunolabeled fibers turned laterally and run towards the subependymal zone.

A dense plexus of axons forming principal SP-immunopositivity occupied the intermediate and dorsal zones of the lateral septum located just above of the ENK-immunoreactive plexus zone (Figure 1B). Wholly, three SP-immunoreactive subfields, two vertically and one horizontally oriented, creating together an inverted U-shaped figure, were distinguished (Figure 2B). The densest accumulation of the SP-containing axons was situated in the lateral vertical subfield (IV) situated along the ventricular wall near the lumen of the lateral ventricle. The horizontal $(\mathrm{H})$ and the medial vertical $(\mathrm{mV})$ subfields appeared to be less densely packed by SP-containing immunoreactive fibers and terminals (Figure 2B).

The TH-immunolabeled principal innervation field was represented by a number of TH-immunolabeled fiber clusters arranged into a prolonged perpendicular formation running over the whole septum in the medial half of the intermediate lateral septal nucleus, i.e. medially from the ENK and SP agglomerations and laterally from the middle line of the septum (Figures 1A, D). The medial part of the $\mathrm{TH}$ formation was more compactly arranged than the lateral one. In the lateral part of the TH agglomeration, many of the TH-immunoreactive profiles imitated triadically branched formations. Generally, ventral and dorsal subfields, marked off in the half of the structure, were distinguished in the TH principal formation. In addition, a small group of axons containing immunoreactivity for TH separated from the main $\mathrm{TH}$ formation, was also regularly seen near the ventricle wall at the middle level of the lateral ventricle. In addition, many individual TH-positive axons were scattered throughout the entire septum and formed dense pericellular terminal baskets around the septal neurons.

Effect of neuroleptics on c-Fos expression. The single injection of VEH had no significant effect on the c-Fos expression occurring in neurons distributed over the whole septum territory (not shown).

The single administration of OLA induced extensive c-Fos expression over the whole septal territory. Many c-Fos particles were accumulated inside of the ENK principal innervation field but large amount of c-Fos profiles occurred also in the surrounding area (Figure 3). In each of the 4 subfields of the ENK principal innervation field heavily, sparse or no innervated c-Fos cells regularly occurred (Figure 3 ).

The OLA treatment also activated cells in the SP-immunolabeled innervation field. Many of c-Fos cells were free of SP-immunoreactive fibers, but several c-Fos cells revealed rich SP-positive 


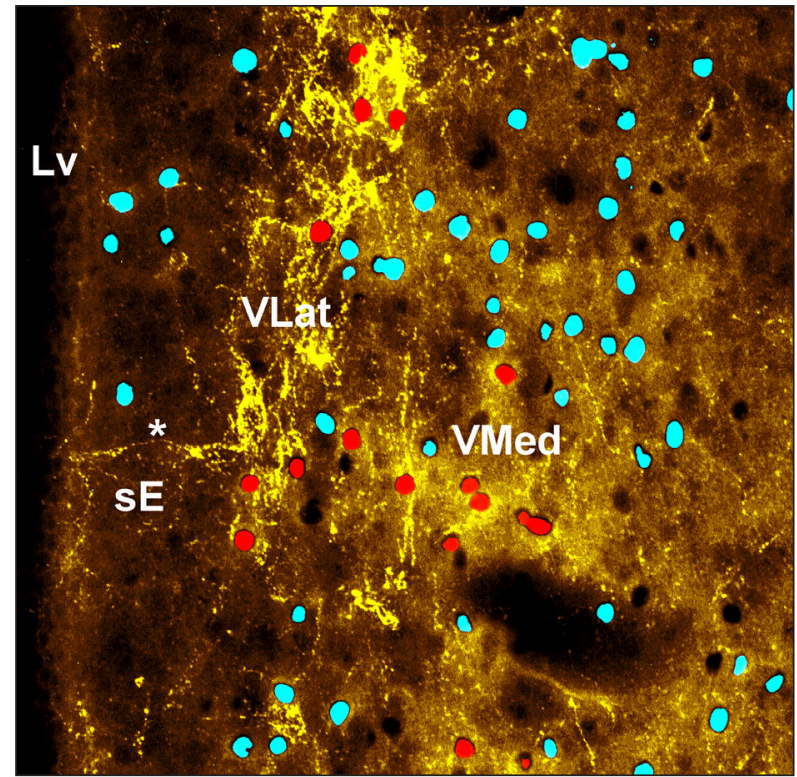

Figure 3. Histological picture illustrating the c-Fos distribution in the VLat and LMed enkephalinergic (ENK) subfields after treatment with olanzapine. Red circles indicate dense and blue circles sparse or no innervation (yellow) of the activated septal cells. A single ENK projection (asterisk) directed to the ependyma is seen in the sE zone. Abbreviations: Lv - lateral ventricle; VLat - ventrolateral subfield; VMed - ventromedial subfield; sE - subependymal zone.

fiber wrapping (Figure 4). The SP-coated c-Fos cells were often present in the lateral vertical SP subfield (Figure 4).

The effect of OLA on the c-Fos expression was very pronounced in the ventral TH principal innervation field including the space between the ENK field ventral portion and the dorsal margin of the accumbens nucleus shell (Figure 5).

The single injection of AMI induced a wideranging c-Fos expression over the whole septum with denser accumulation in its ventral part at the level of the borderline with the shell of the accumbens nucleus (Figure 6A). However, inside of the ENK principal innervation field less amount of c-Fos profiles occurred than in the surrounding area. The c-Fos profiles were evenly distributed over the whole ENK innervation field and were present in each of the 4 ENK-labeled subfields (Figure 6B). c-Fos cells anatomical relationship to the ENK innervation profiles could be divided into three levels: heavily and completely coated forming the typical basket figures, partially coated by fiber arbors (Figure 6C) or not touched with the ENK fibers or terminals.

The treatment with AMI activated many cells in the SP-labeled subfields, mainly in the vertical one

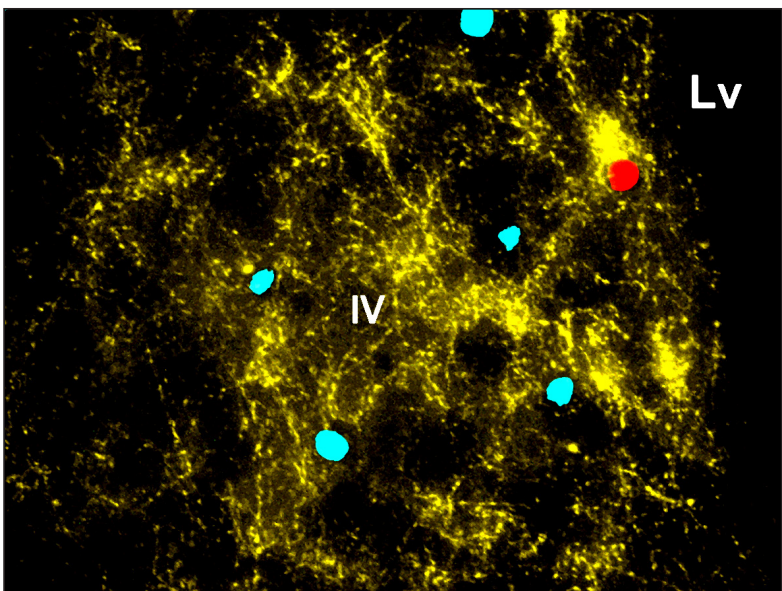

Figure 4. Histological picture demonstrating one heavily (red circle) and several uninnervated (blue circles) c-Fos-displaying cells after treatment with olanzapine in the lV subfield of the SP principal innervation field. Abbreviations: $\mathrm{Lv}$ - lateral ventricle; lV - lateral vertical SP subfield.

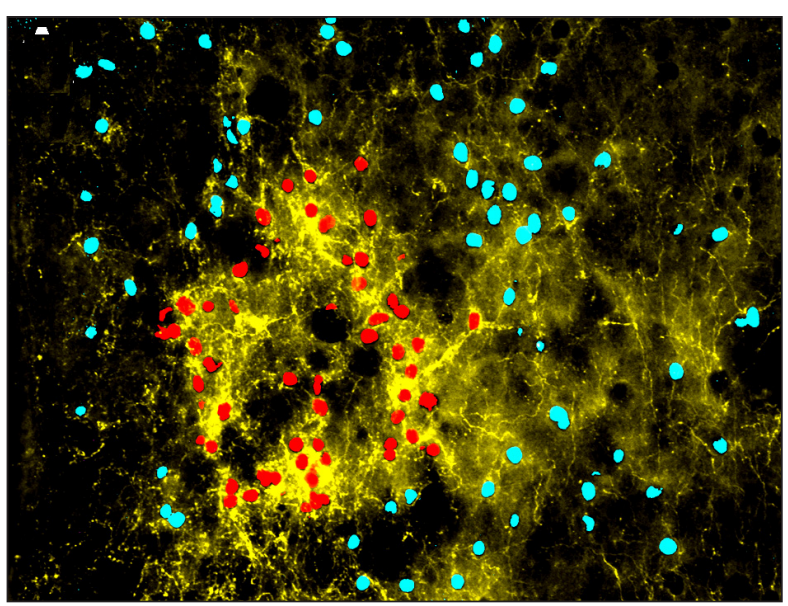

Figure 5. Demonstration of c-Fos distribution around (blue circles) and within (red circles) the tyrosine hydroxylase (TH)labeled groups of cells located near the accumbens nucleus shell dorsal margin. Almost all of the c-Fos-labeled cells (red circles) are heavily coated by TH processes (yellow). The surrounding c-Fos-labeled cells (blue circles) are less densely or uninnervated by $\mathrm{TH}$ immunoreactive fibers (yellow).

located along the lateral ventricle (Figure 7A). In this SP subfield, many of the cells with c-Fos occurrence were tightly surrounded/coated by the SP-positive cell processes (Figure 7B). Within this area, however, some of the c-Fos exhibiting cells revealed only a sparse SP innervation cover.

The AMI administration also activated c-Fos expression in the TH principal innervation field. The majority of the c-Fos expressing cells occurred in the 


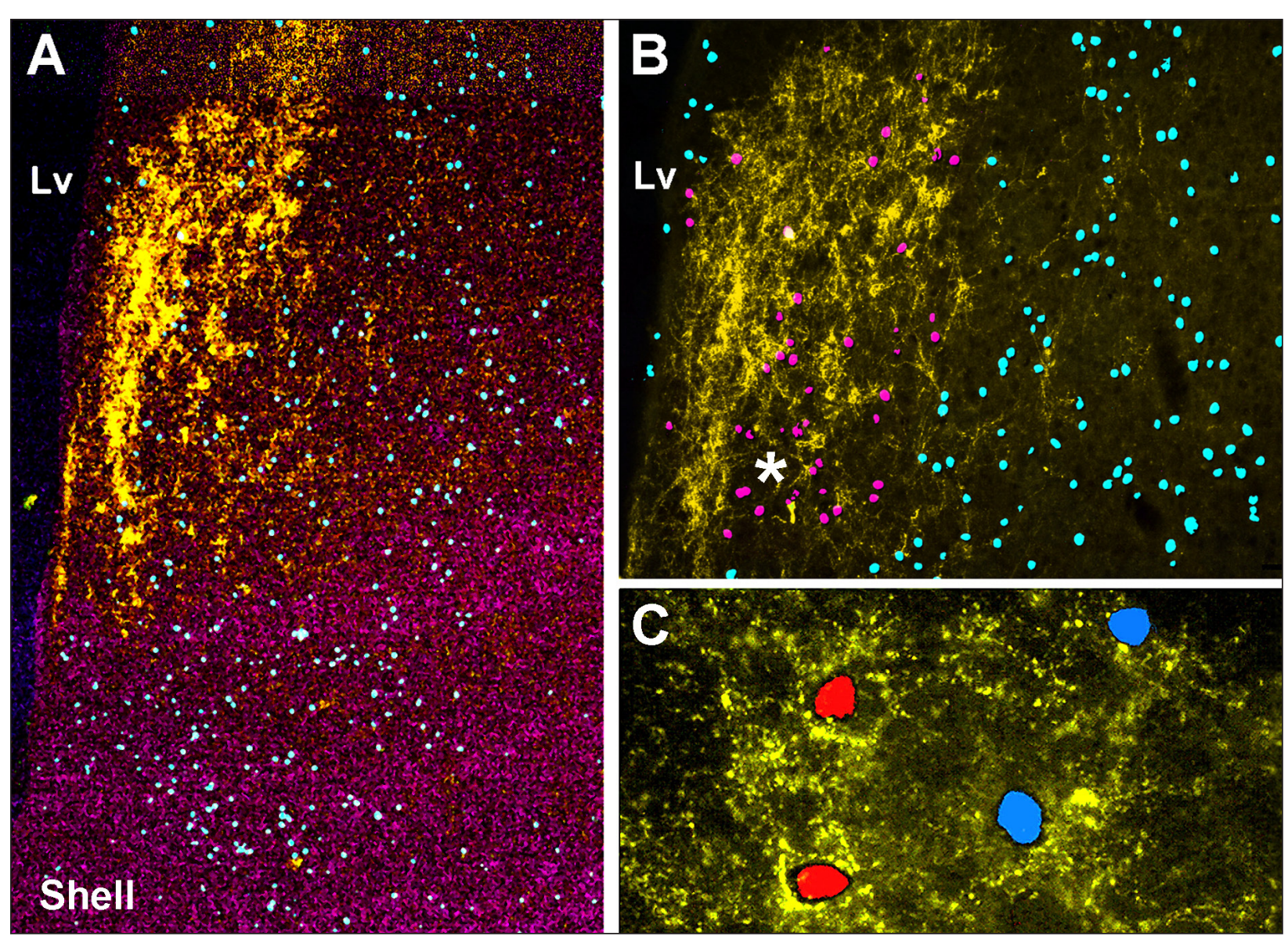

Figure 6. Illustration of c-Fos distribution (blue circles)) within and around the principal enkephalinergic (ENK) innervation field after amisulpride administration in the ventral and intermedial lateral septum (A). More detailed view (B) on the ENK area magnified from the A picture indicating less occupancy of the ENK principal field with c-Fos profiles (red circles) than the surrounding area (blue circles). Most c-Fos profiles are seen in the VMed subfield (asterisk) of the ENK principal field. C-Fos profiles localized within the pericellular baskets (C) of activated cells (red circles) abutting with several sparsely innervated cells (blue circles). Abbreviations: $\mathrm{Lv}$ - lateral ventricle; shell - accumbens $\mathrm{n}$. shell.

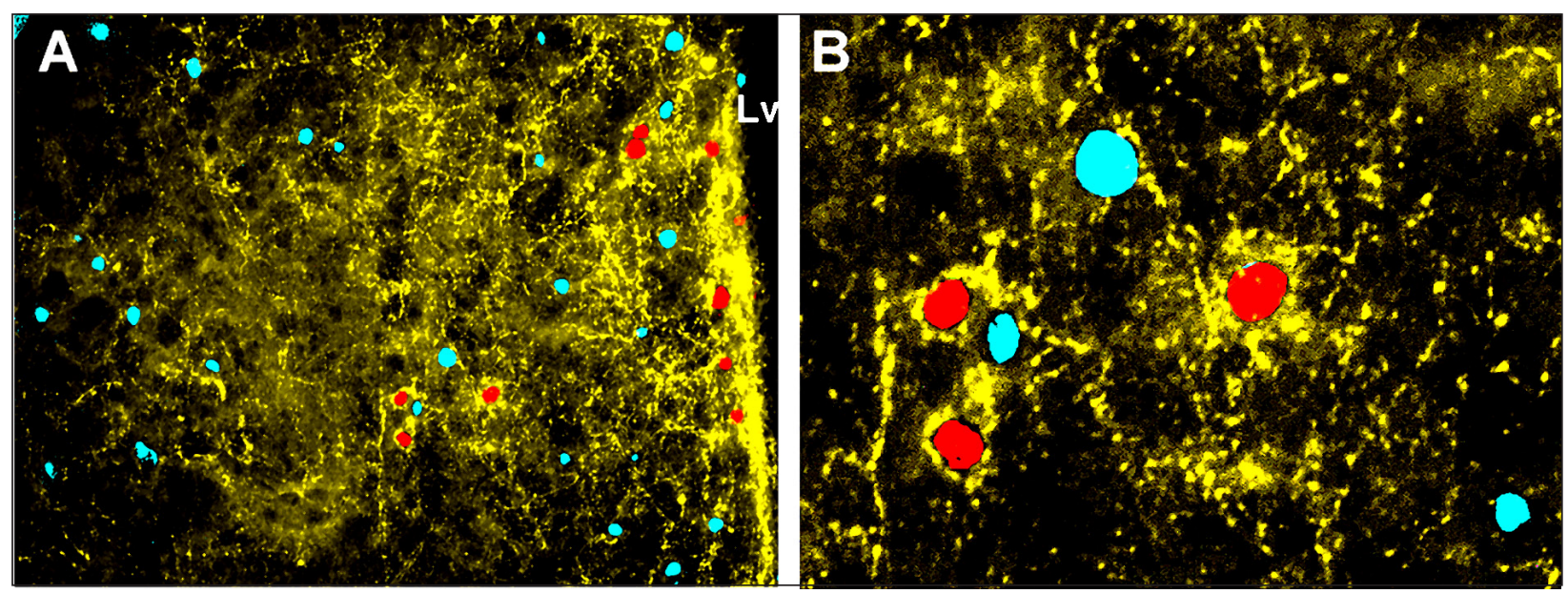

Figure 7. Distribution of c-Fos displaying cells after amisulpride treatment in the $\mathrm{lV}$ and $\mathrm{mV}$ subfields of the substance $\mathrm{P}$ (SP) principal field (A). Detailed view (B) on a group of c-Fos profiles located within (red circles) and outside (blue circles) of the pericellular baskets of activated cells. Abbreviations: $\mathrm{Lv}$ - lateral ventricle; $\mathrm{lV}$ - lateral vertical; $\mathrm{mV}$ - medial vertical. 
lateral subfield of the TH main agglomeration, where many of the TH-immunoreactive profiles imitated from triadically to multiradically branched formations (Figure 8A). The cells displaying c-Fos presence were regularly surrounded by the TH-immunoreactive profiles (Figure $8 \mathrm{~B}$ ).
The single administration of QUE induced an extensive c-Fos expression over the whole septum. Densest accumulation of c-Fos profiles occurred in the ventral lateral portion of the septum just above the dorsal margin of the shell of the accumbens nucleus (Figure 9A). The principal ENK innervation
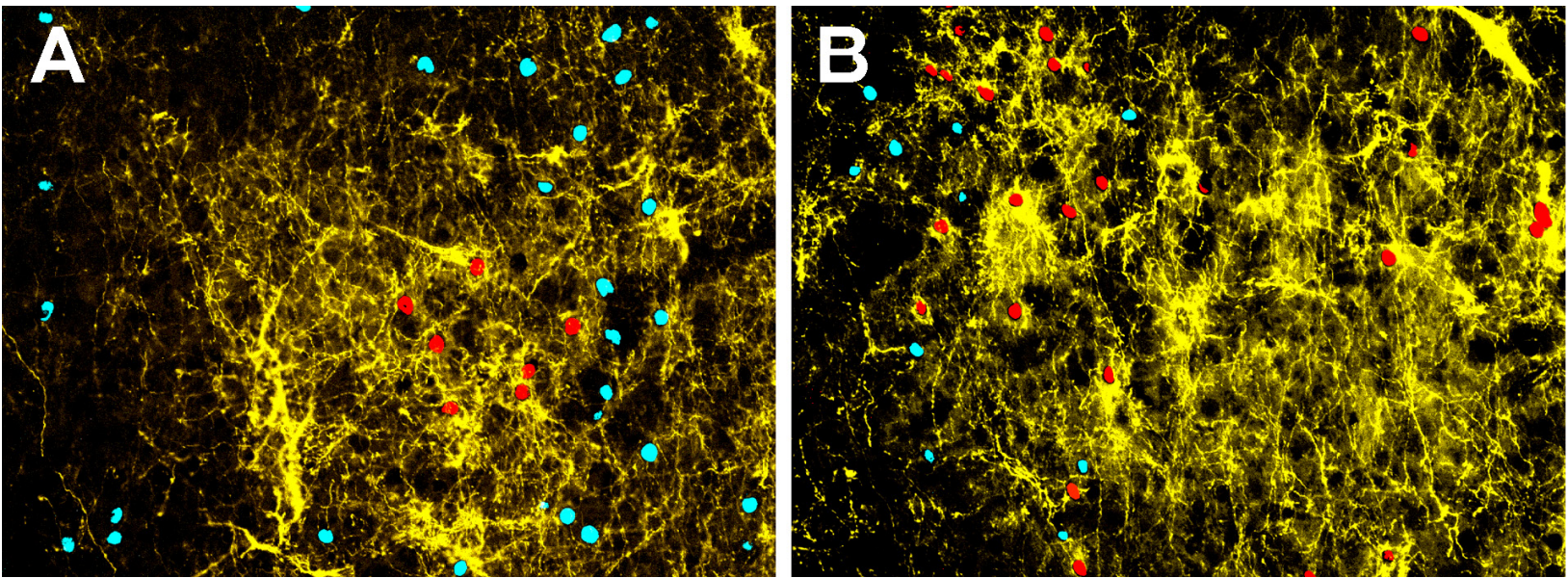

Figure 8. Distribution of c-Fos displaying cells after amisulpride treatment in the dorsal subfield of the tyrosine hydroxylase (TH) principal innervation field (A). In the TH-immunoreactive island, many of the activated cells (red circles) are heavily surrounded by TH immunoreactive axons and terminals (yellow), but distinct number of them is also free of this innervation (blue circles). In the ventral subfield of the TH principal innervation field (B) many of the activated cells displays c-Fos profiles located within (red circles) than outside (blue circles) of the pericellular baskets of activated cells.
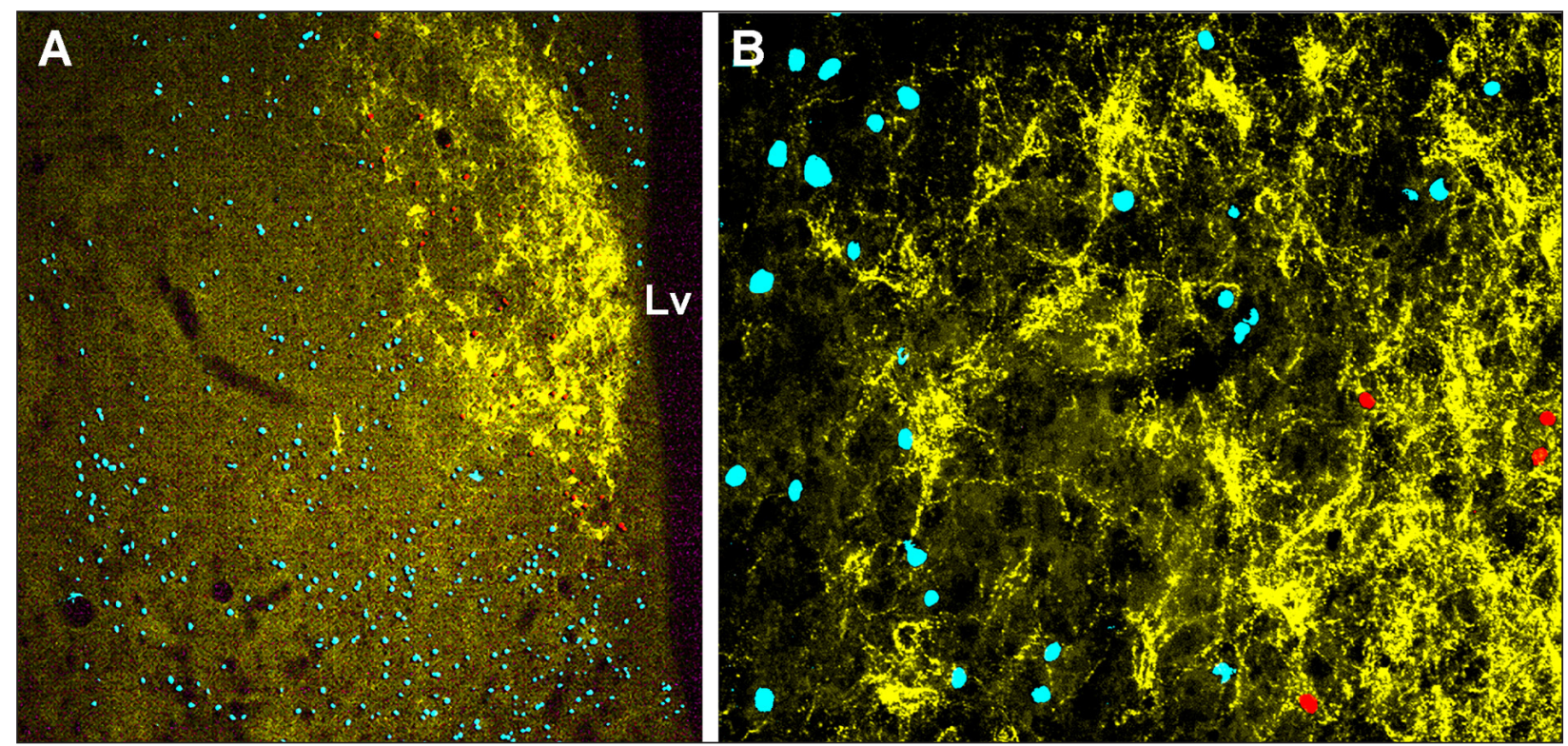

Figure 9. Distribution of c-Fos displaying cells (blue circles) around the enkephalinergic (ENK) principal innervation field (yellow) after quetiapine treatment in the septum (A). The presence of c-Fos profiles in the ENK principal field (red circles) is lower than in the surrounding septal area (A). Many of the c-Fos containing cells (red circles) are densely surrounded by ENK processes (B). A distinct population of c-Fos cells (blue circles) is also free of ENK occupancy. Abbreviations: Lv - lateral ventricle. 
field was surrounded by a number of c-Fos particles. However, in comparison with the c-Fos presence in the near vicinity of the ENK field, each of the four ENK subfields was only slightly occupied by c-Fos profiles (Figure 9B). Within the whole ENK principal innervation field, great variations occurred in the c-Fos profiles innervation density, some of them were densely packed by ENK terminals, while others displayed sparser or no ENK innervation (Figure 9B).

The QUE injection also induced c-Fos expression in cells located in each of the three SP-labeled subfields (Figure 10). The stimulatory effect of QUE was quite expressive in each of the SP subfields, where many of the c-Fos labeled cells were massively ringed with SP-positive axons and terminals (Figures 10B, D). Many of the c-Fos profiles containing cells were tightly surrounded/coated with a dense accumulation of SP-immunolabeled fibers and terminals (Figures 10B, D).

The administration of QUE also activated a number of c-Fos expressed cells in the TH-immunolabeled field (Figure 11A). Some of the c-Fos cells were tightly surrounded/coated by QUE-positive triadically or more than triadically branched processes (Figures 11A, B). Even in the small group of TH-labeled profiles accumulated in the periventricular zone, c-Fos cells densely surrounded by TH-labeled processes were detected (Figure 11B).

The ARI induced c-Fos expression almost homogeneously dispersed over the whole septum. Likewise, in the case of the other antipsychotics, ARI also stimulated c-Fos expression in the ENK prin-
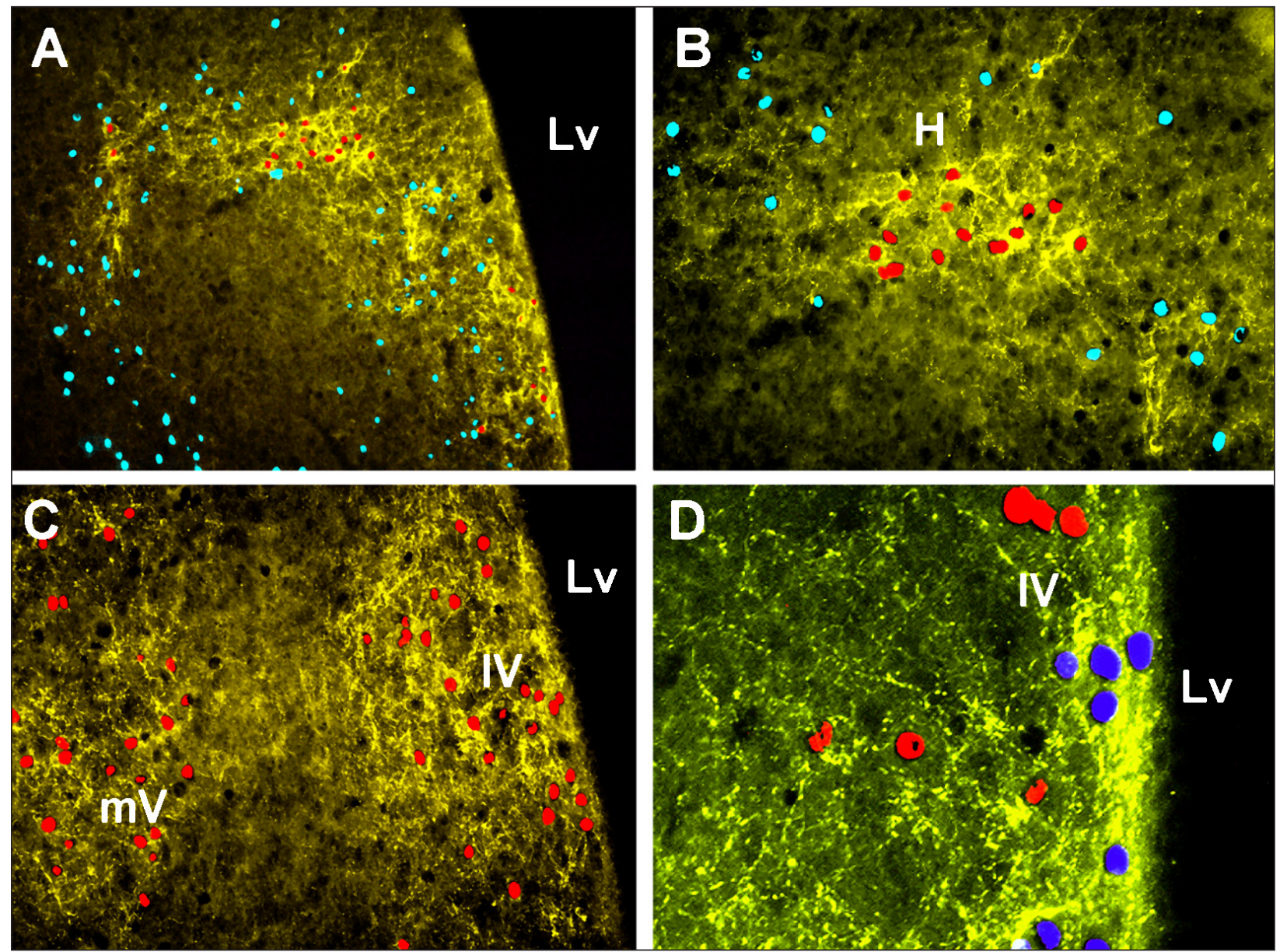

Figure 10. Distribution of c-Fos displaying cells within the substance $\mathrm{P}(\mathrm{SP})$ principal innervation field after quetiapine treatment in the septum. The whole SP innervation field (A) is surrounded by a large number of c-Fos profiles (blue circles). A distinct population of stimulated cells exhibiting c-Fos is present in all three SP subdivisions (B, C, D). Detailed view on the densely (B, C - red circles and D - blue circles) and sparsely (D - red circles) innervated c-Fos-containing cells in the lV SP innervation subfield (yellow). Abbreviations: $\mathrm{IV}$ - lateral vertical subfield; $\mathrm{mV}$ - medial vertical subfield; $\mathrm{H}$ - horizontal subfield; $\mathrm{Lv}$ - lateral ventricle. 


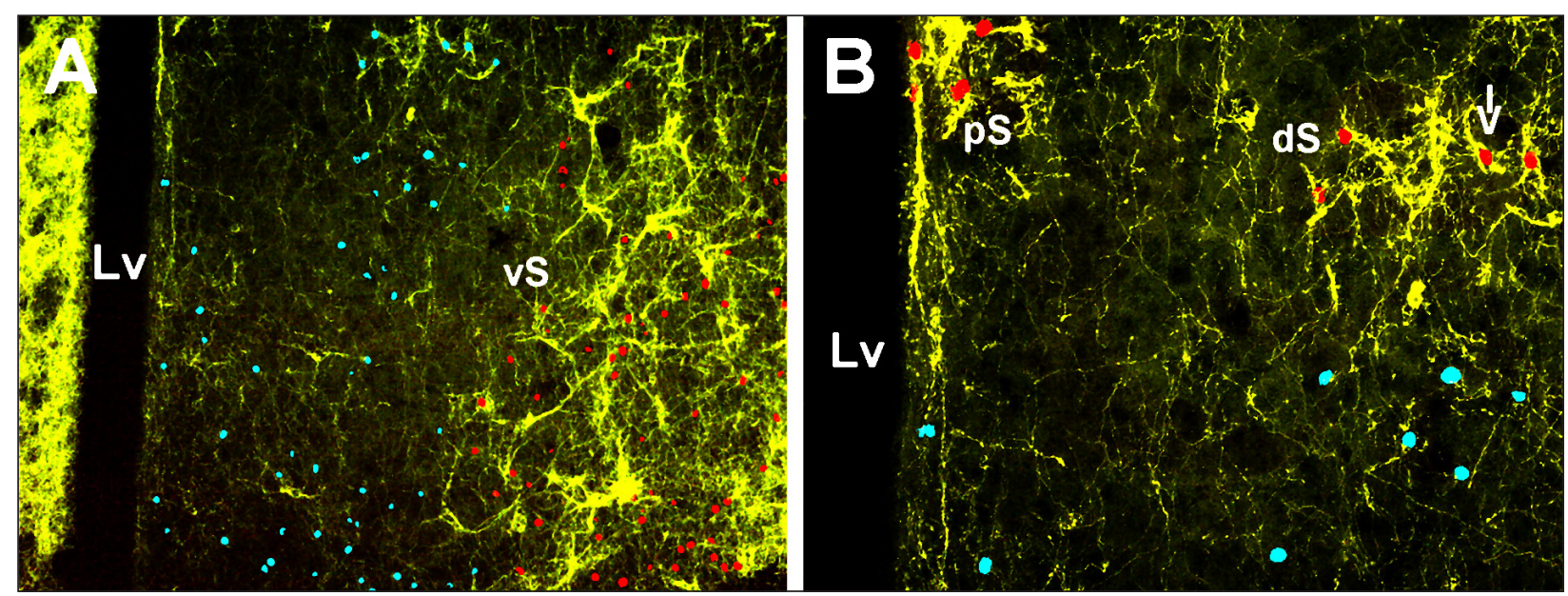

Figure 11. Distribution of c-Fos displaying cells within the tyrosine hydroxylase (TH) vS, dS, and pS principal innervation fields after quetiapine treatment in the septum. The TH vS field (A) is heavily accumulated with c-Fos profiles of activated cells (red circles) in comparison with the surrounding area (blue circles). Many of the activated cells are densely coated by TH axons and terminals (arrow) also in the TH dS (B) as well as in the TH pS (B) principal innervation subfields. Abbreviations: Lv - lateral ventricle; vS ventral vertical subfield; dS - dorsal medially twisted subfield; pS - periventricular subfield.

cipal immunoreactive fiber agglomeration. The c-Fos profiles were distributed over the whole ENK-labeled area, however, in the dorso- and ventro-lateral subfields their occurrence appeared poorer. Actually, two population of c-Fos expressing cells regularly occurred in the ENK principal area, one that was heavily coated by ENK processes and one that was sparsely or not coated with ENK fibers (Figure 12).

The treatment with ARI also induced c-Fos expression in the entire SP principal innervation field with the most abundant occurrence in the lateral vertical subfield along the wall of the lateral ventricle (Figure 13). The majority of c-Fos cells was only partially touched with the SP-innervation and only small population of them showed rich coating with SP fibers (Figure 13).

The ARI-induced c-Fos expression in TH-immunoreactive principal field was not evidently rich and the occurrence of c-Fos cells was very poor in each of the TH principal field two subfields. Usually, only partially TH-coated c-Fos expressing cells were observable. The occurrence of densely TH coated c-Fos cells was rare (not shown).

\section{Discussion}

The data of the present study indicate that OLA AMI, QUE, and ARI single treatment differently activate c-Fos expression in cells located in the dissimilar ENK, SP, and TH innervation fields of the rat septum.

The septal complex forms an interface between the limbic telencephalic structures associated with cognition and motivation, on the one side, and hypothalamic and brain stem areas related to endocrine and autonomic functions maintaining the balance between the endocrine and emotional components of the CNS, on the other side (Jakab and Leranth 1993). Each of the antipsychotic drugs activated only a few cells over the whole ENK principal innervation field, however, many of them did not show tight contact with the ENK-immunolabeled processes. In addition, the number of c-Fos-bearing cells in each of the antipsychotic action was obviously lower in the ENK principal field than in the surrounding area. The origin of the ENK-ergic innervation of the rat lateral septal nuclei has been shown to lay in 4 discrete brain regions: the perifornical hypothalamic area, the anterior hypothalamic nucleus, the bed nucleus of the stria terminalis, and the dorsal hypothalamic area (Onteniente et al. 1989; Sakanaka and Magari 1989). However, the anatomical configuration of ENK pathways within the septum ENK principal innervation field has not been exactly delineated yet. Thus, the multiple origin of ENK innervation of the septum implies that these ENK axons with different origin may have different functions, which clearly indicate that the functional involvement of septal cells in the ENK area activated by the individual antipsychotics might be very different.

The stimulatory effect of QUE on c-Fos expression was most expressive from all the neuroleptics investigated in each of the SP subfields analyzed. In 
addition, many of the c-Fos labeled cells were richly surrounded by SP-positive axons and terminals. AMI also induced c-Fos expression in cells heavily surrounded by SP axons and terminals mainly in the SP lateral vertical subfield. Some of the terminals coated the c-Fos labeled cells appeared as liketerminal bouttons. Actually, it has been demonstrated by the preembedding immunoelectron microscopy (Morita et al. 1991) that the SP axonal terminals frequently make synapses with neuronal somata and small dendrites in the vicinity of the neuronal perikarya in the lateral septum. Therefore, it is reasonable to believe that from all the antipsychotics studied, the QUE and AMI might most markedly contribute to the functional complexity of SP actions in the septum, for example in the facilitation of memory formation and learning (Staubli and Huston 1980; Khakpai et al. 2013). Likewise in the case of ENK, the majority of SP-like immunoreactive fibers in the lateral septal area has been shown to be supplied by SP cells from more sources located in the area between the anterior hypothalamic nucleus and the lateral hypothalamus and in the latero-dorsal tegmental nucleus (Sakanaka et al. 1982), which indicate that the functional involvement of the activated septal cells in the SP innervated area by the individual antipsychotics may also be very dissimilar. There exist also assumptions that since neurons in the lateral septal area receive hippocampal input and project massively to hypothalamic areas, the different types of SP inputs on these neurons may modify the information flow arriving from the hippocampus to diencephalic brain structures at the level of the lateral septal area (Varoqueaux and Leranth 1997). However, whether by antipsychotics activated cells heavily innervated by SP axons and terminals might be a part of the above mentioned hippocampal-diencephalic circuit must be assessed by further studies.

The septal region also receives midbrain dopamine innervations originating in the ventral tegmental area (Oades and Halliday 1987; Adams and Moghaddam 2000). These innervations make direct synaptic contacts with perikarya and dendrites of the lateral septal neurons producing both excitatory and inhibitory postsynaptic responses (Adams and Moghaddam 2000). Ascending adrenergic axons originating in the locus coeruleus also supply the septum with catecholaminergic fibers (HaghdoostYazdi et al. 2009). The findings of the present work corroborate the literature data that catecholaminergic innervation of the septum is very rich consisting of the TH principal innervation field and smaller periventricular TH-positive fibers network and

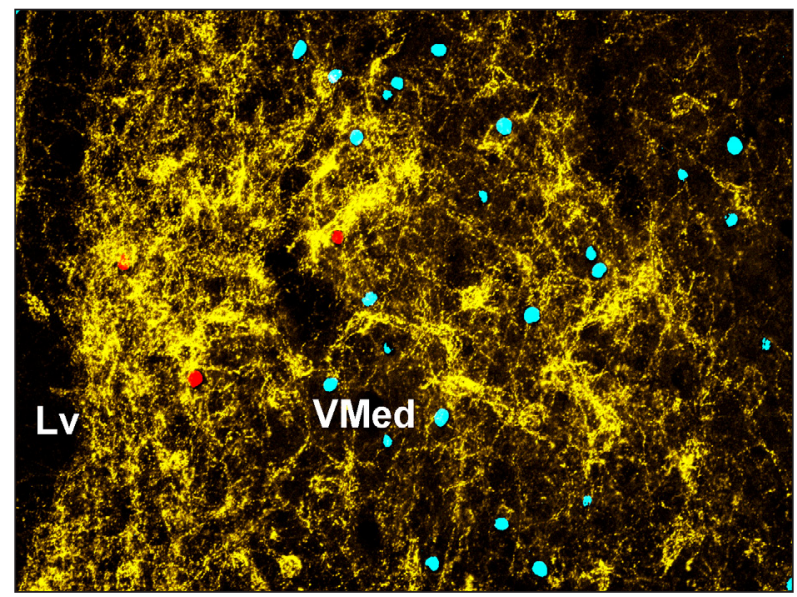

Figure 12. Distribution of c-Fos displaying cells within the enkephalinergic (ENK) principal innervation field after aripiprazole treatment in the septum. The c-Fos profiles located within the ENK VLat subfield are only lightly occupied by c-Fos profiles. Only small number of the activated cells (red circles) displays dense infoldings formed by ENK immunoreactive profiles (yellow). The majority of them (blue circles) is free of the heavy innervation. Abbreviations: VMed - ventromedial subfield; Lv - lateral ventricle.



Figure 13. Distribution of c-Fos displaying cells within the substance P (SP) IV subfield after aripiprazole treatment in the septum. Only small number of the activated cells displays dense infoldings (red circles) formed by SP immunoreactive profiles (yellow). The majority of them (blue circles) is free of the heavy innervation coating. Abbreviations: IV - lateral vertical subfield; $\mathrm{Lv}$ - lateral ventricle.

many TH-labeled triadically or more than triadically branched processes dispersed over the whole septum. All the neuroleptics used induced c-Fos staining in the TH-immunolabeled principal innervation field. However, their effect was variable. The positive effect of OLA on c-Fos expression was distinct in the TH 
ventral vertical subfield and area below, i.e. near the accumbens nucleus shell dorsal margin. AMI activated also septal cells occupying the dorsal subfield of the TH principal innervation field and QUE activated also cells located in the TH periventricular subfield. This regionally dispersed action of neuroleptics clearly indicate that the effect of the individual antipsychotics is markedly different within the septum, which probably may also stand for the functional heterogeneity in their actions.

Taken together, the data of the present study indicate that ENK, SP, and TH innervation fields might influence separate populations of septal cells acti- vated by AMI, OLA, QUE, and ARI antipsychotics and that each of these region-differently innervated cells might also be associated with the functional heterogeneity of the individual lateral septal nuclei.

\section{Acknowledgement}

The authors are grateful for the c-Fos antibody (12-5) donated by Dr. Jens D. Mikkelsen (Denmark) and financial support provided by $2 / 0037 / 19$ VEGA grant of the Slovak Academy of Sciences and APVV15-0037 grant of the Research and Development Agency of the Slovak Republic.

\section{References}

Adams BW, Moghaddam B. Tactile stimulation activates dopamine release in the lateral septum. Brain Res 858, 177-180, 2000.

de Bartolomeis A, Marmo F, Buonaguro EF, Rossi R, Tomasetti C, Iasevoli F. Imaging brain gene expression profiles by antipsychotics: region-specific action of amisulpride on postsynaptic density transcripts compared to haloperidol. Eur Neuropsychopharmacol 23, 1516-1529, 2013.

de Bartolomeis A, Tomasetti C, Iasevoli F. Update on the mechanism of action of aripiprazole: translational insights into antipsychotic strategies beyond dopamine receptor antagonism. CNS Drugs 29, 773-799, 2015.

DeVries GJ, Buijs RM. The origin of the vasopressinergic and oxytocinergic innervation of the rat brain with special reference to the lateral septum. Brain Res 273, 307-317, 1983.

Gall C, Moore RY. Distribution of enkephalin, substance P, tyrosine hydroxylase, and 5-hydroxytriptamine immunoreactivity in the septal region of the rat. J Comp Neurol 225, 212-227, 1984.

Goodnick PJ, Jerry JM. Aripiprazole: profile on efficacy and safety. Expert Opin Pharmacother 3, 1773-1781, 2002.

Grady MA, Gasperoni TL, Kirkpatrick P. Aripiprazole. Nar Rev Drug Discov 2, 427-428, 2003.

Haghdoost-Yazdi H, Pasbakhsh P, Vatanparast J, Rajaei F, Behzadi G. Topographical and quantitative distribution of the projecting neurons to main divisions of the septal area. Neurol Res 31, 503-513, 2009.

Hokfelt T, Skirboll L, Rehfeld JF, Goldstein M, Markey K, Dann O. A subpopulation of mesencephalic dopamine neurons projecting to limbic areas contains a cholecystokinin-like peptide: evidence from immunohistochemistry combined with ret-rograde tracing. Neurosci 5, 2093-2124, 1980.

Ishikawa K, Inoue K, Tosaka H, Shimada O, Suzuki M. Immunohistochemical characterization of thyrotropin-releasing hormone-containing neurons in rat septum. Neuroendocrinology 39, 448-452, 1984.

Jakab RL, Leranth C. Presence of somatostatin or neurotensin in lateral septal dopaminergic axon terminals of distinct hypothalamic and midbrain origins: convergence on the somatospiny neurons. Exp Brain Res 92, 420-430, 1993.

Khakpai F, Nasehi M, Haeri-Rohani A, Eidi A, Zarrindast MR. Septo-hippocampo-septal loop and memory formation. Basic Clin Neurosci 4, 5-23, 2013.

Kohler C, Eriksson LG. An immunohistochemical study of somatostatin and neurotensin positive neurons in the septal nuclei of the rat brain. Anat Embryol (Berl) 170, 1-10, 1984.

MacGibbon GA, Lawlor PA, Bravo R, Dragunow M. Clozapine and haloperidol produce a differential pattern of immediate early gene expression in rat caudate-putamen, nucleus accumbens, lateral septum and islands of Calleja. Mol Brain Res 23, 21-32, 1994.

Mailman RB, Murthy V. Third generation antipsychotic drugs: partial agonism or receptor functional selectivity? Curr Pharm Des 16, 488-501, 2010.

Majercikova Z, Cernackova A, Horvathova L, Osacka J, Pecenak J, Kiss A. Effect of acute asenapine treatment on Fos expression in frontal brain structures under normal conditions and mild stress preconditioning in rat. Brain Res Bull 108, 60-66, 2014.

Mandal N, Singh OP, Sen S. Extrapyramidal side effects with low doses of amisulpride. Ind J Psych 56, 197-199, 2014. 
Morita F, Sakanaka M, Magari S, Sakai H. Fine structure of substance P-containing nerve fibers in the rat lateral septum; simultaneous localization in pre- and postsynaptic elements, Neurosci Lett 122, 99-102, 1991.

Natesan S, Reckless GE, Nobrega JN, Fletcher PJ, Kapur S. Dissociation between in vivo occupancy and functional antagonism of dopamine D2 receptors: comparing aripiprazole to other antipsychotics in animal models. Neuropsychopharmacology 31, 1854-1863, 2006.

Natesan S, Reckless GE, Barlow KBL, Nobrega JN, Kapur S. Amisulpride the 'atypical' atypical antipsychotic - Comparison tohaloperidol, risperidone and clozapine. Schizophrenia Res 105, 224-235, 2008.

Natesan S, Reckless GE, Barlow KB, Nobrega JN, Kapur S. Partial agonists in schizophrenia--why some work and others do not: insights from preclinical animal models. Int J Neuropsychopharmacol 14, 1165-1178, 2011.

Nemeroff CB, Kinkead B, Goldstein J. Quetiapine: preclinical studies, pharmacokinetics, drug interactions, and dosing. J Clin Psychiatry 63 (Suppl 13), 5-11, 2002.

Nomikos GG, Tham CS, Fibiger HC, Svensson TH. The puta in rat medial prefrontal cortex and lateral tive atypical antipsychotic drug amperozide preferentially increases c-fos expression septum. Neuropsychopharmacology 17, 197-201, 1997.

Oades RD, Halliday GM. Ventral tegmental (A10) system: neurobiology. 1. Anatomy and connectivity. Brain Res Rev 12, 117-165, 1987.

Onteniente B, Menetrey D, Arai R, Calas A. Origin of the met-enkephalinergic innervation of the lateral septum in the rat. Cell Tiss Res 256, 585-592, 1989.

Ozdemir V, Fourie J, Ozdener F. Aripiprazole (Otsuka Pharmaceutical Co). Curr Opin Investig Drugs 3, 113-120, 2002.

Paxinos G, Watson C. The rat brain in stereotaxic coordinates. Academic Press 1997.

Risold PY, Swanson LW. Chemoarchitecture of the rat lateral septal nucleus. Brain Res Rev 24, 91-113, 1997.

Robertson GS, Fibiger HC. Effects of olanzapine on regional c-Fos expression in rat forebrain. Neuropsychopharmacology 14, 105-110, 1996.

Sakanaka M, Shiosaka S, Takatsuki K, Inagaki S, Hara Y, Kawai Y, Senba E, Tohyama M. Origins of substance Pcontaining fibers in the lateral septal area of young rats: immunohistochemical analysis of experimental manipulations. J Comp Neurol 212, 268-277, 1982.

Sakanaka M, Magari S, Emson PC, Hillyard CJ, Girgis SI, MacIntire I, Tohyama M. The calcitonin gene-related peptide-containing fiber projection from the hypothalamus to the lat-eral septal area including its fine structure. Brain Res 344, 196-199, 1985.

Sakanaka M, Magari S, Shibasaki T, Lederis K. Corticotropin releasing factor-containing afferents to the lateral septum of the rat brain. J Comp Neurol 270, 404-413, 1988.

Sakanaka M, Magari S. Reassessment of enkephalin ENK-containing afferents to the rat lateral septum with reference to the fine structures of septal ENK fibers. Brain Res 479, 205-216, 1989.

Sebens JB, Koch T, Ter Horst GJ, Korf J. Olanzapine-induced Fos expression in the rat forebrain; cross-tolerance with haloperidol and clozapine. Eur J Pharmacol 353, 13-21, 1998.

Schoemaker H, Claustre Y, Fage D, Rouquier L, Chergui K, Curet O, Oblin A, Gonon F, Carter C, Benavides J, Scatton B. Neurochemical characteristics of amisulpride, an atypical dopamine D2/D3 receptor antagonist with both presynaptic and limbic selectivity. J Pharmacol Exp Ther 280, 83-97, 1997.

Staubli U, Huston JP. Facilitation of learning by post-trial injection of substantia P into the medial septal nucleus. Behav Brain Res 1, 245-255, 1980.

Swanson LW, Cowan WM. The connections of the septal region in the rat. J Comp Neurol 186, 621-656, 1979.

Swanson LW, Risold PY. On the Basic Architecture of the Septal Region. In: Numan R. (eds) The Behavioral Neuroscience of the Septal Region. Springer, New York, NY, 2000.

Swainston-Harrison T, Perry CM. Aripiprazole: a review of its use in schizophrenia and schizoaffective disorder. Drugs 64, 1715-1736, 2004.

Tamminga CA, Kane JM. Olanzapine (Zyprexa): characteristics of a new antipsychotic. Expert Opin Investig Drugs 6, 1743-1752, 1997.

Varoqueaux F, Leranth C. Hypothalamo-septal enkephalinergic fibers terminate on AMPA receptor-containing neurons in the rat lateral septal area. Synapse 25, 263-271, 1997.

Wan W, Ennulat DJ, Cohen BM. Acute administration of typical and atypical antipsychotic drugs induces distinctive patterns of Fos expression in the rat forebrain. Brain Res 688, 95-104, 1995.

Winans E. Aripiprazole. Am J Health Syst Pharm 60, 2437-2445, 2003. 